\title{
Marketing digital como una estrategia y publicidad para promover la agricultura y ganadería en la parroquia Moraspungo del Cantón Pangua.
}

\section{Digital Marketing as a strategy and publicity to promote agriculture and livestock in the parish of the Canton Pangua Moraspungo.}

Velasteguí López Efraín. ${ }^{1}$, Zambrano Guerrero Natali ${ }^{2}$

\section{Resumen.}

DOI: $\underline{\text { https://doi.org/10.33262/visionariodigital.v1i3.244 }}$

El presente ensayo tiene como finalidad conocer las actividades agropecuarias en una de las fuentes de desarrollo del país, las estrategias de marketing son los surcos donde sembrar un plan de acciones para alcanzar visibilidad y tráfico en internet. Con mayor trascendencia en economías donde importantes porcentajes de la población se vinculan a esta, pues se cuenta actualmente con los medios para diseñar y evaluar estrategias que lleven a la implementación de mecanismos de apoyo al sector agropecuario.

La importancia del marketing, sobre todo lo primero es saber que existen una serie de procesos organizados, en pro de un objetivo y para cubrir una necesidad es marketing. Y por ello, sin vinculación ninguna a una empresa.

Las estrategias consisten en un conjunto de acciones que se llevan adelante para alcanzar determinados objetivos y metas relacionadas al sector agropecuario, el cual se ha transformado en una prioridad para todas aquellas pequeñas, medianas y grandes empresas que quieran ser competitivas en el mercado.

Tener una buena estrategia de marketing digital en redes sociales es un objetivo fundamental, las mismas que se han descubierto en los ecosistemas social media el cual se han transformado en una ventana que posibilita a los usuarios seguir de cerca a celebridades y expertos.

Al igual que la agricultura y ganadería son actividades económicas de origen antiguo que día a día se viene desarrollando en la crianza de animales y siembra de plantas silvestres, medicinales y hortalizas entre otras para su aprovechamiento. En la actividad agropecuaria de la crianza de animales consiste en la obtención de varios productos leche, carne, huevos, pieles entre otros Palabras claves: Marketing digital, publicidad, agricultura, ganadería.

\footnotetext{
${ }^{1}$ Universidad Técnica de Ambato, Ambato, Ecuador, le.velastegui@uta.edu.ec ${ }^{2}$ Universidad Técnica de Cotopaxi Extensión la Maná, Ecuador, zambranon@ gmail.com 


\begin{abstract}
.
The purpose of this essay is to know the agricultural activities in the sources of the development of the country, the marketing strategies and the action plans to reach visibility and traffic on the internet. With greater importance in the links where the percentages of the population are found, they are linked to this, as well as the means to evaluate and evaluate support strategies for the agricultural sector.

The importance of marketing, especially the first thing is to know that there is a series of organized processes, in favor of an objective and to cover a need is marketing. And for that reason, without any connection to a company ..

The strategies consist of a set of actions that are sustained so that the objectives and the agricultural sector are met, which becomes a priority for all small, medium and large companies that want to be competitive in the market.

Having a good digital marketing strategy in social networks is a fundamental goal, the networks that have been discovered in social networking systems, which has become a window that enables users to follow celebrities and experts.

As agriculture and livestock are economic activities of ancient origin that day by day is included in the raising of animals and planting wild plants, medicinal and vegetables among others for their use. In the agricultural activity of raising animals consists of obtaining several products milk, meat, eggs, cakes and others
\end{abstract}

Keywords: Digital marketing, advertising, agriculture, livestock.

\title{
Introducción.
}

Recuperar los saberes locales y reentender como se logra producir sin atentar contra el medio, son los inicios del mejoramiento de la calidad de vida de los campesinos. El mundo va evolucionando y con él la tecnología, la cual nos permite desarrollar nuevas formas en el medio de comunicación, mediantes estas innovaciones es el llamado Marketing Digital.

Marketing digital es la aplicación de estrategias de comercialización llevadas a cabo en los medios digitales que nos permiten nuevas herramientas, está caracterizado por la combinación y utilización de estrategias de comercialización en medios digitales, la cual nace con la posibilidad de compartir información ha través de las redes sociales y nuevas tecnologías de información.

Su principal riqueza es la agricultura y ganadería son dos actividades económicas estratégicas para la sociedad y es fundamental para ayudar a mantener la zona rural vivo, esta parroquia permite el desarrollo de las zonas rurales, contribuyendo economía y socialmente al bienestar de su población.

La agricultura actual es productiva y respetuosa con el medio ambiente. Dentro de este sector ha permitido maximizar la eficiencia de las producciones garantizando al mismo tiempo, 
destacándose la producción del café, cacao, banano, caña de azúcar, yuca y cítricos en grandes cantidades, la ganadería llegando a ser también una fuente de ingresos para sus moradores.

\section{Desarrollo.}

¿Qué es marketing digital?

Thompson, Techopedia \& Chris (2018) aseguran que el marketing digital la finalidad de las empresas u organizaciones es mantenerse enfocado en los diferentes segmentos del marketing para así poder obtener clientes, mediantes las vías tecnologías con cuya finalidad mantenerse comunicados en todo momento con los clientes y brindarles un mejor servicio.

Mediante mi criterio estoy de acuerdo con los autores, que de tal manera el marketing digital aportan un valor de vida de las personas a través de las plataformas digitales, con una información real de acuerdo a lo enmendado en la búsqueda lo cual se nos hace más fácil obtener la información gracias a la tecnología que va evolucionando día a día, es por ello que el marketing encontró nuevas formas y valiosas herramientas el cual le permite comunicarse y brindar servicios a sus clientes es de entonces que nace el marketing digital, ya que contamos con la dicha de vivir en la era de estar conectados, la principal función del marketing digital es estar conectados con sus clientes las 24 horas del día y los 7 días de la semana.

Gráfico N 1: Componentes y resultados del concepto de marketing.

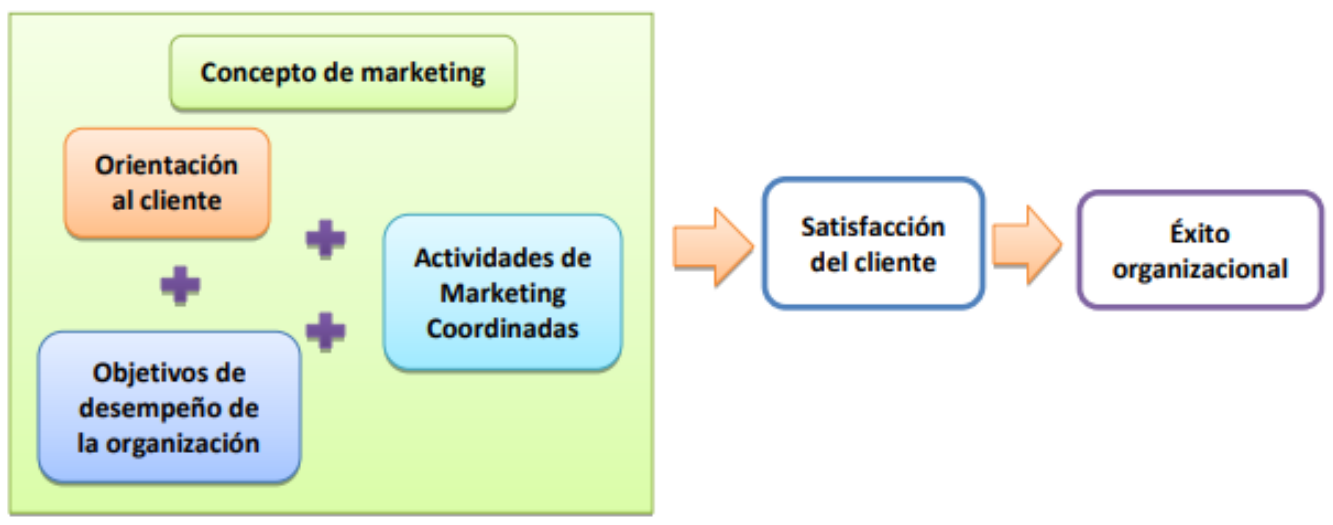

Fuente: Stanton, Etzel y Walker, "Fundamentos de Marketing".

\section{Marketing estratégico.}

Cerrajeria (s.f) señala que el marketing estratégico es utilizado como un análisis sistemático y continuado de las características del mercado y del desarrollo de conceptos o productos rentables, los cuales también están orientados hacia grupos de consumidores determinados, teniendo en cuenta la competencia y procurando alcanzar una ventaja competitiva defendible a largo plazo. 
En mi criterio personal analizando la información, estoy de acuerdo con el autor en el cual el marketing estratégico esta activamente con la orientación y formulación de las estrategias de la empresa donde nos facilita la información, sobre la evolución de las nuevas estrategias de la segmentación del mercado.

¿Qué es publicidad?

Stanton (s.f) \& Guinn (s.f) afirman que la publicidad es un esfuerzo pagado el cual es trasmitido por medios de la información con el objetivo de informar, la publicidad consiste en la colocación de avisos y mensajes, en tiempo o espacio pagado por un patrocinador claramente identificado promoviendo ideas para los anuncios de la plataforma.

Según mi criterio personal mutuamente de acuerdo con los 3 autores, ya que la publicidad es una forma de comunicación entre la empresa y cliente, el cual le permite un largo alcance de información, de igual manera la publicidad va creciendo día a día con nuevas expectativas mediante la tecnología y esto hace que sea más fácil publicarlo en una plataforma digital y dejar atrás los anuncios en papeles volantes, carteles entre otros.

\section{El cantón Pangua.}

INEC, (2010) El Cantón Pangua, en la Provincia de Cotopaxi, en el centro del Ecuador, se formó el 1 de junio de 1938, su cabecera cantonal es la ciudad de El Corazón.

Pangua conocida como el "Paraíso Escondido de Cotopaxi". Se encuentra ubicada al Sur Occidente de la provincia, en las estribaciones Occidentales de la Cordillera Andina, a una altitud que va desde los 100 a 3.600 m.s.n.m por sus 8 pisos altitudinales, con una temperatura que va desde los $10^{\circ} \mathrm{C}$ en su zona alta y de 20 a $25^{\circ} \mathrm{C}$ en su zona baja. Su nombre viene de la cultura Cayapa es por eso Pangua=Panguapi que significa "Agua Nueva", los pánguense han construido su identidad y reconocimiento de ser un pueblo noble y valiente, hospitalario y trabajador, creando lazos de hermandad entre los demás cantones de la Provincia.

La mayoría de las parroquias de este cantón tienen gran producción de productos tanto de la sierra como de la costa, allí se da la afamada panela, donde existen numerosos alambiques (trapiches) con gran producción de aguardiente (la sabrosa "bananita"), madera, frutas y demás. El cantón ha progresado enormemente en los últimos años, es por ello que la Municipalidad pone en conocimiento su desarrollo en las diferentes áreas unas de ellas el turismo que es fundamental para la economía del cantón por lo que invitan a conocer de este maravilloso paraje natural y cultural.

Límites.

Norte, la Maná, Pílalo, Pujili.

Sur, con el rió Piñanatug que divide a la Provincia de Cotopaxi con Bolívar. 
Este, Angamarca, Pujili.

Oeste, Quinsaloma, Ventanas, Quevedo.

División política.

\section{Parroquias urbanas}

$\checkmark$ El Corazón (cabecera cantonal)

$\checkmark$ Parroquias rurales

$\checkmark$ Moraspungo

$\checkmark$ Ramón Campaña

$\checkmark$ Pinllopata

Características.

Clima: El cantón presenta varios climas: templado en su zona urbana, frío en la región alta y cálida en áreas del Subtrópico.

Flora: La flora existente está constituida especialmente de árboles de eucalipto en asociación con pinos. El espacio restante se hace la práctica de cultivos en menor escala ya que su suelo se encuentra erosionado por el uso de agroquímicos.

Fauna: Algunos animales mamíferos también se puede observar como lobos, cervicabra, pumas, venados, caballos salvajes, toros bravos, alpacas, conejos, osos entre otros. Los pájaros que viven en la zona son los imponentes Cóndores andinos, gigles, gavilán de espalda roja, curiquingue, águilas, torcaza, gaviota andina, perdiz de páramo, gallareta, pato punteado y quilico.

Parroquia Moraspungo

Límites: La parroquia Moraspungo está ubicada en el sector oeste del Cantón Pangua Limitada al norte con los cantones La Mana y Pujilí, al Sur con la provincia de Bolívar y al Este con la provincia de los Ríos.

Altura: la parroquia Moraspungo se encuentra a 360 metros sobre el nivel del mar.

Superficie: 44000 hectáreas.

Clima: Subtrópico.

Gráfico N2: Participación en la producción total de cultivos permanentes

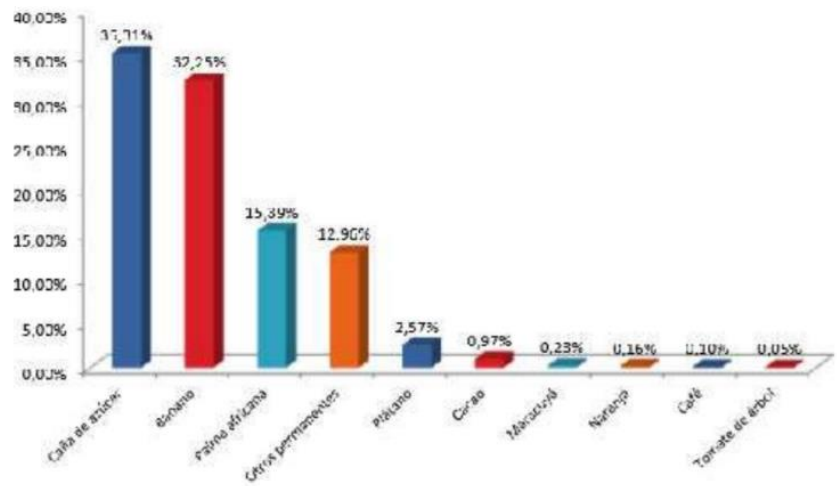

Fuente: Encuesta de Superficie y Producción Agropecuaria Continua (ESPAC) 2011, del INEC

¿Qué es la agricultura y ganadería? 
Ikerd (s.f) afirma que la agricultura son procesos biológicos como una posibilidad para eficientizar la producción, son sistemas integrados con prácticas de producción de planta y animal en un lugar específico, el cual satisface las necesidades humanas de alimento y fibras. Con el objetivo de mantener una vialidad económica en las operaciones de la unidad de producción y elevar la calidad de vida del productor y la sociedad en general.

Aledit (s.f) señala que ganadería son actividades económicas de origen antiguo, esta se desarrolla en la crianza de animales y la obtención de varios productos como carne, pieles, leche, huevos etc.

En mi criterio personal podría decir que la ganadería y agricultura están relacionadas, ya que en este caso los ganados en un rancho o granja aportan el estiércol, esto se lo puede utilizar como abono en el cultivo, alimentos para los animales y para la siembra en los invernaderos. Estas dos actividades humanas dependen del tipo especial de medio ambiente, que es el medio rural del agro ecosistema.

Cotopaxi representa el 3,88\% de la superficie de labor agrícola a nivel nacional. El banano es su cultivo de mayor producción.

\begin{tabular}{|c|c|c|c|c|c|c|}
\hline \multicolumn{7}{|c|}{ Cultivos permanentes de mayor producción } \\
\hline Cultiv & manentes & Super & e plantada (Ha) & Superficie co & chada (Ha) & Producción (TM.) \\
\hline \multicolumn{2}{|c|}{ Banano } & & 3.138 & \multicolumn{2}{|c|}{3.015} & 83.456 \\
\hline \multicolumn{2}{|c|}{ Plátano } & & 7.445 & \multicolumn{2}{|c|}{6.815} & 18.287 \\
\hline \multicolumn{2}{|c|}{ Otros } & & 20.702 & \multicolumn{2}{|c|}{18.215} & 15.493 \\
\hline \multicolumn{7}{|c|}{ Cultivos transitorios de mayor producción } \\
\hline Cult & & Super & e plantada (Ha) & Superficie co & chada $(\mathrm{Ha})$ & Producción (TM.) \\
\hline \multicolumn{2}{|c|}{ Papa } & & 11.033 & \multicolumn{2}{|c|}{9.818} & 64.190 \\
\hline \multicolumn{2}{|c|}{ Tomate riñón } & & 125 & \multicolumn{2}{|c|}{125} & 6.491 \\
\hline \multicolumn{2}{|c|}{ Otros } & & 53.360 & \multicolumn{2}{|c|}{47.120} & 29.254 \\
\hline \multicolumn{7}{|c|}{ Número total de cabezas (machos y hembras) } \\
\hline Vacuno & Porcino & Ovino & Asnal & Caballar & Mular & Caprino \\
\hline 272.109 & 118.708 & 195.380 & 13.533 & 11.299 & 4.525 & 2.383 \\
\hline
\end{tabular}

Fuente: Encuesta de Superficie y Producción Agropecuaria Continua (ESPAC) 2010, del INEC

\section{A nivel del país.}

En el sector pecuario predomina el ganado vacuno con un total de 5,3 millones de cabezas, seguido por el porcino con 1,5 millones de cabezas. 

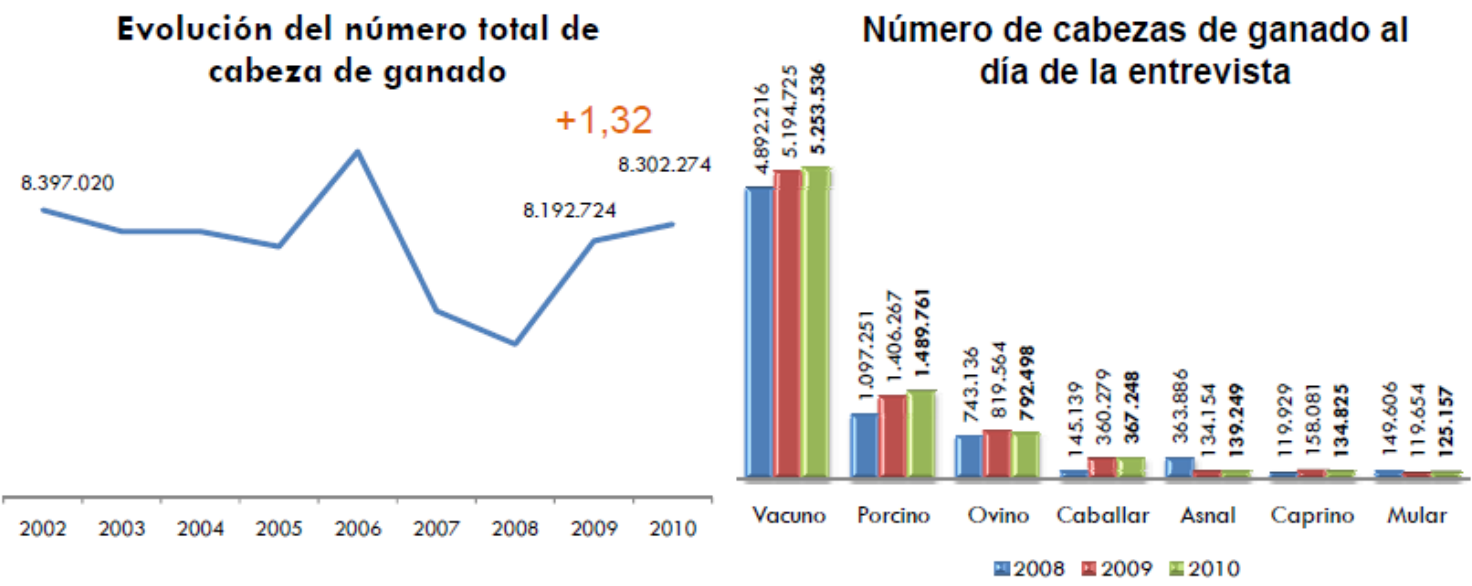

Fuente: Encuesta de Superficie y Producción Agropecuaria Continua (ESPAC) 2010, del INEC

La producción diaria de leche se incrementó en 9,19\% entre 2009 y 2010. En la Sierra más de 702 mil vacas fueron ordeñadas, $64,5 \%$ del total nacional.

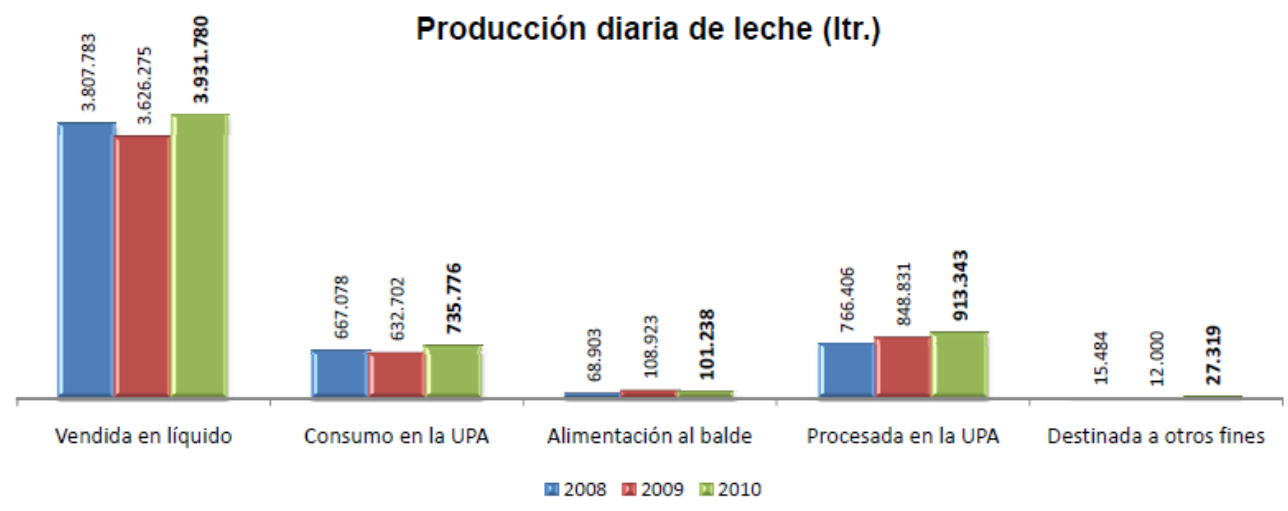

Fuente: Encuesta de Superficie y Producción Agropecuaria

Continua (ESPAC) 2010, del INEC 
La existencia de aves criadas en el campo se incrementó un 4,8\% entre 2009 y 2010

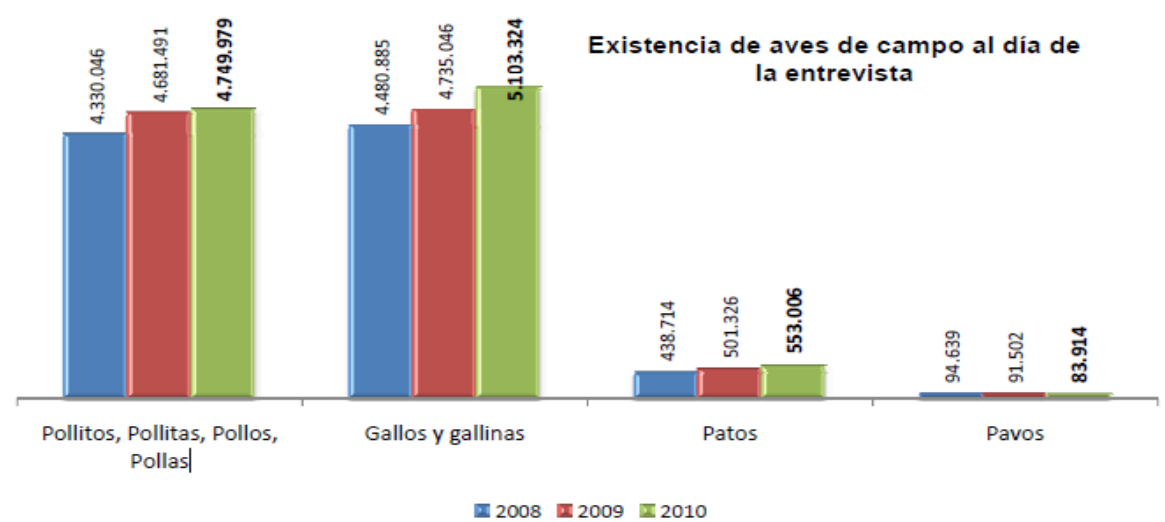

Fuente: Encuesta de Superficie y Producción Agropecuaria Continua (ESPAC) 2010, del INEC

La existencia de aves criadas en planteles avícolas se incrementó un 1,66\% entre 2009 y 2010

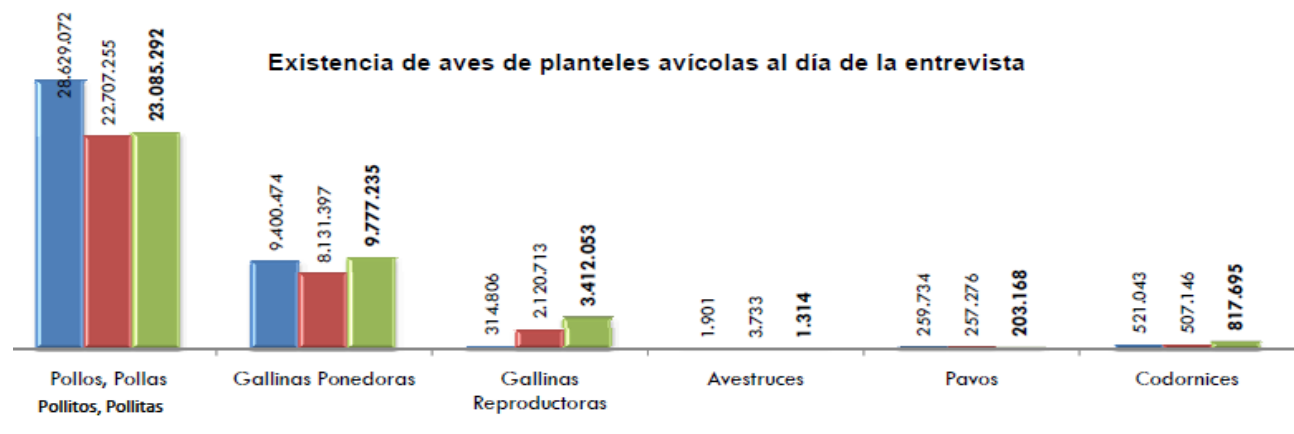

Fuente: Encuesta de Superficie y Producción Agropecuaria Continua (ESPAC) 2010, del INEC

Provincias con mediana superficie agropecuaria

\begin{tabular}{|c|c|c|c|c|c|c|}
\hline \multirow[b]{2}{*}{ Provincia } & \multicolumn{4}{|c|}{ Superficie de labor agropecuaria (Ha) } & \multirow[b]{2}{*}{ Total } & \multirow[b]{2}{*}{$\begin{array}{l}\text { Participación } \\
\text { macional }\end{array}$} \\
\hline & $\begin{array}{c}\text { Cullivos } \\
\text { permanentes }\end{array}$ & $\begin{array}{l}\text { Cultivos } \\
\text { Transitorios y } \\
\text { Barbecho }\end{array}$ & $\begin{array}{c}\text { Pastos } \\
\text { Culltivados }\end{array}$ & $\begin{array}{c}\text { Pastos } \\
\text { Naturales }\end{array}$ & & \\
\hline PICHINCHA & 51.354 & 26.962 & 187.742 & 111.982 & 378.040 & $5,27 \%$ \\
\hline EL ORO & 86.786 & 8.428 & 228.925 & 14.160 & 338.299 & $4,72 \%$ \\
\hline AZUAY & 5.987 & 36.609 & 85.221 & 181.791 & 312.608 & $4,36 \%$ \\
\hline COTOPAXI & 37.439 & 81.938 & 123.039 & 63.920 & 306.336 & $4,27 \%$ \\
\hline BOLIVAR & 38.806 & 34.503 & 138.333 & 79.660 & 291.302 & $4,06 \%$ \\
\hline CHIMBORAZO & 2.109 & 69.000 & $\$ 2.532$ & 110.699 & 264.340 & $3,69 \%$ \\
\hline
\end{tabular}

FUENTE: Encuesta de Superficie y Producción Agropecuaria Continua (ESPAC) 2011, del INEC 


\section{Conclusiones.}

- Al terminar nuestra investigación podemos determinar, que el marketing ha entrado al mundo de la globalización internacional, lo que ha provocado que las innovaciones tecnológicas, hayan tenido una aplicación y desarrollo para hacer negocios y la publicidad de los productos.

- Lo que está bien claro es que las estrategias de marketing digital con más futuro son aquellas que ofrecen algo más al usuario, un valor agregado, más que una simple publicidad.

- En el marketing digital ayudaría en gran medida a las medianas empresas, ya que es una forma de publicidad económica y de rápida propagación. Sus beneficios pueden ser vistos a corto plazo y permite la medición de respuestas de parte del público objetivo.

- La falta de conocimiento e inversión en tecnología es una barrera que no permite que las medianas empresas se den a conocer en medios web, ya que la mayoría no cuenta con página web ni correo electrónico y no ven necesaria la publicidad en Internet.

\section{Bibliografía.}

Aledit, B. P. (s.f.). La Ganaderia. Agroindustria definiciones y realidades.

Asociation, A. M. (s.f.). La publicidad. Fundamentos del marketing.

Cerrageria, L. M. (s.f.). Padre de Marketing.

Chris, A. (2018). Que es el marketing digital. SEO.

Ikerd, J. E. (s.f.). Agricultural Economics.

O’Guinn Tomas, A. C. (s.f.). Publicidad. International Thomson, 6.

Stanton William, E. M. (s.f.). La publicidad. Fundamentos de marketing, 569.

Techopedia. (2018). Que es el marketing digital. SEO marketing digital.

Thompson, I. (2018). Que es el marketing. SEO marketing digital.

\section{Ciencia
Digital
Edtacal}




\section{Para citar el artículo indexado.}

Velasteguí E. \& Zambrano N. (2017). Marketing digital como una estrategia y publicidad para promover la agricultura y ganadería en la parroquia Moraspungo del Cantón Pangua. Revista electrónica Visionario Digital 1(1), 5-14. Recuperado desde: http://cienciadigital.org/revistacienciadigital2/index.php/VisionarioDigital/article/view/244/5 $\underline{46}$

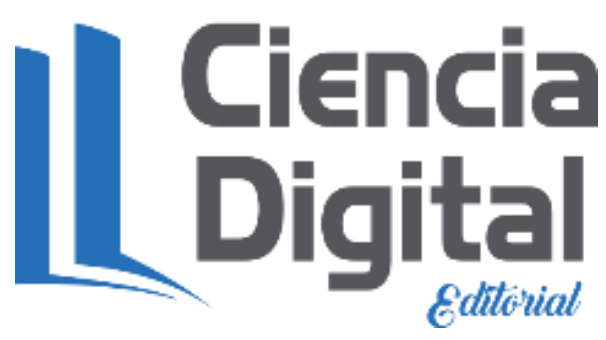

El artículo que se publica es de exclusiva responsabilidad de los autores y no necesariamente reflejan el pensamiento de la Revista Ciencia Digital.

El articulo queda en propiedad de la revista y, por tanto, su publicación parcial y/o total en otro medio tiene que ser autorizado por el director de la Revista Ciencia Digital
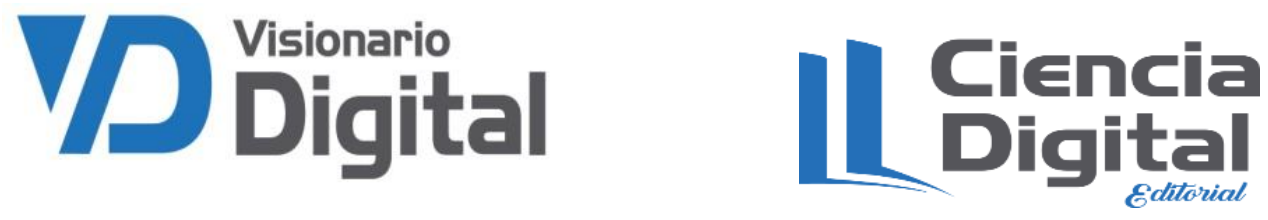